\title{
A mathematical model of solute dynamics in a curved artery
}

\author{
G. Pontrelli ${ }^{1} \&$ A. Tatone ${ }^{2}$ \\ ${ }^{1}$ Istituto per le Applicazioni del Calcolo, CNR, Roma, Italy \\ ${ }^{2}$ DISAT, Facoltà di Ingegneria, Università dell'Aquila, Italy
}

\begin{abstract}
A mathematical model for the mass transport and diffusion process in arteries is presented. Blood flow is described by the unsteady Navier-Stokes equation, and solute dynamics by an advection-diffusion equation. A linearization procedure over the steady state solution is carried out and an asymptotic analysis is used to study the influence of a small curvature with respect to the straight tube. Some numerical experiments in cases of physiological interest are presented: the results show the characteristics of the long wave propagation and the role played by the geometry on the solute distribution.

Keywords: solute dynamics, arterial transport, curved tubes, advection-diffusion equation, numerical methods.
\end{abstract}

\section{Introduction}

Mass transport and diffusion phenomena inside the arterial lumen and through the vascular wall are of great importance for physiological functions, such as oxygenation, nourishment of tissues and metabolic processes. Some mathematical models coupling 3D flow and solute dynamics have been developed in recent years [1-4]. They are defined in a finite arterial segment of arbitrary shape, where an inflow solute distribution is provided [1,2]. Some of them consider also absorption and exchange through the vascular tissues [3]. All these models provide the local concentration pattern and are useful to understand the relationship between the local flow pattern, the nourishment of arterial tissues and possible pathologies derived when such a process is altered [4].

On the other hand, fluid dynamical factors such as flow separation, recirculation and wall shear stress are correlated to the atherosclerotic lesions. It is known 
that these phenomena tend to occur in regions of branching or in bending sites, where flow axisymmetry is lost. In particular geometrical effects, such as curvature, strongly affect the flow pattern and consequently the concentration of gases and substances dissolved in the blood [5]. Moreover, mass transfer phenomena are intrinsically coupled with the hemodynamics which influences the wall permeability and fluid diffusivity.

In a recent work, the oscillating flow through an elastic thin-walled curved artery has been investigated, using an asymptotic analysis based on the assumption of a small curvature parameter [6]. In the present paper, a similar approach is used to model the mass transport and diffusion process inside a straight or moderately curved artery. This is described by the advection-diffusion equation and a Robinlike interface condition is imposed at the boundary to match internal and external fluxes, and with the flow field preassigned. For most substances such a process is convection dominated, due to a low diffusion coefficient [5]. Being interested in propagative phenomena, the solute dynamics inside the vascular tissue is deemed a negligible phenomena and the so called free-wall model is used [4]. Induced by the periodicity of respiratory, hormonal and feeding acts, the concentration of a substance in blood is subject both to an oscillation in time and to a spatial variation along the vessel, sustained by the fluid motion [5]. The wave period is strongly dependent of the substance considered. As a consequence, for any substance, we look for the propagation characteristics, in relation with the medium diffusivity and wall permeability properties. The aim of this study is to characterize the solute propagation in the blood flow and to provide the local distribution of concentration that can be affected by geometrical factors, such as the curvature. Some numerical simulations show the dispersion curve and predict an asymmetric profile for concentration. This can be useful to reveal relationship between local flow patterns and process of altered absorption or anomalous accumulation of substances on the arterial wall.

\section{Formulation of the problem}

The motion of blood in a vessel is modelled by the flow of a newtonian viscous fluid in a cylindrical tube. Different substances are dissolved in blood, transported through the stream and possibly exchanged through the arterial wall [5]. For simplicity, the presence of one solute only is considered and let us denote by $c$ its concentration. Because of both diffusive and convective phenomena, $c$ satisfies the following advection-diffusion equation $[1,7,8]$ :

$$
\frac{\partial c}{\partial t}+\boldsymbol{v} \cdot \nabla c-\nabla \cdot(\mu \nabla c)=0
$$

with $\boldsymbol{v}$ the fluid velocity, $\mu>0$ a diffusivity coefficient. A possible exchange of solute through the wall is expressed by:

$$
(\mu \nabla c) \cdot \boldsymbol{n}+\sigma c=\sigma c_{e x t}
$$


where $\sigma \geq 0$ is the wall permeability and $c_{\text {ext }}$ is an external constant concentration (if the wall is impermeable, $\sigma=0$ ). Strictly speaking, $\mu$ and $\sigma$ do depend on the flow field and on the temperature $[1,2]$ but, for simplicity, let us assume them as constants. Any possible solute chemical reacting effect is also neglected.

Due to the small value of $\mu$, for most substances the problem is convection dominated. For $\mu \rightarrow 0$ the problem changes nature and tends to be purely hyperbolic. Note that the problem (1)-(2) is homogeneous when $c_{e x t}=0$ or when $\sigma=0$ and admits $\infty^{1}$ solutions.

In principle, fluid and solute dynamics are coupled processes and influence reciprocally. However in this model the solute is regarded as a passive scalar: it is simply advected by the blood flow in the lumen, neglecting any feedback effect on the fluid viscosity and density. As consequence, we split the flow problem from the mass transport: the fluid velocity $v$ is computed beforehand, and the problem (1)-(2) is subsequently solved.

Let us decompose the variables $\boldsymbol{v}$ and $c$ as sum of a steady state part (denoted with a bar) and an unsteady component (indicated with a circumflex accent):

$$
\boldsymbol{v}=\overline{\boldsymbol{v}}+\hat{\boldsymbol{v}} \quad c=\bar{c}+\hat{c}
$$

and let us assume the unsteady parts $\hat{\boldsymbol{v}}$ and $\hat{c}$ (and also $\nabla \hat{\boldsymbol{v}}$ and $\nabla \hat{c}$ ) are small enough with respect to the steady ones such that the nonlinear term $\boldsymbol{v} \cdot \nabla c$ in eqns. (1) can be linearized as:

$$
(\overline{\boldsymbol{v}}+\hat{\boldsymbol{v}}) \cdot(\nabla \bar{c}+\nabla \hat{c}) \approx \overline{\boldsymbol{v}} \cdot \nabla \bar{c}+\overline{\boldsymbol{v}} \cdot \nabla \hat{c}+\hat{\boldsymbol{v}} \cdot \nabla \bar{c}
$$

neglecting the higher order terms.

The steady state solution satisfies the following boundary value problem:

$$
\begin{aligned}
& \overline{\boldsymbol{v}} \cdot \nabla \bar{c}-\mu \nabla^{2} \bar{c}=0 \\
& \mu \nabla \bar{c} \cdot \boldsymbol{n}+\sigma \bar{c}=\sigma c_{\text {ext }} \quad \text { at the wall }
\end{aligned}
$$

and admits the solution:

$$
\bar{c}=c_{\text {ext }} \quad \text { if } \sigma \neq 0
$$

and

$$
\bar{c}=\text { const } \quad \text { if } \sigma=0
$$

This corresponds to the fact that, for a time long enough, the solute pervades the whole tube and, at infinite time, reaches a uniform concentration. Because no further hypothesis has been made, the steady solution results independent either of the domain geometry and of the velocity field. 


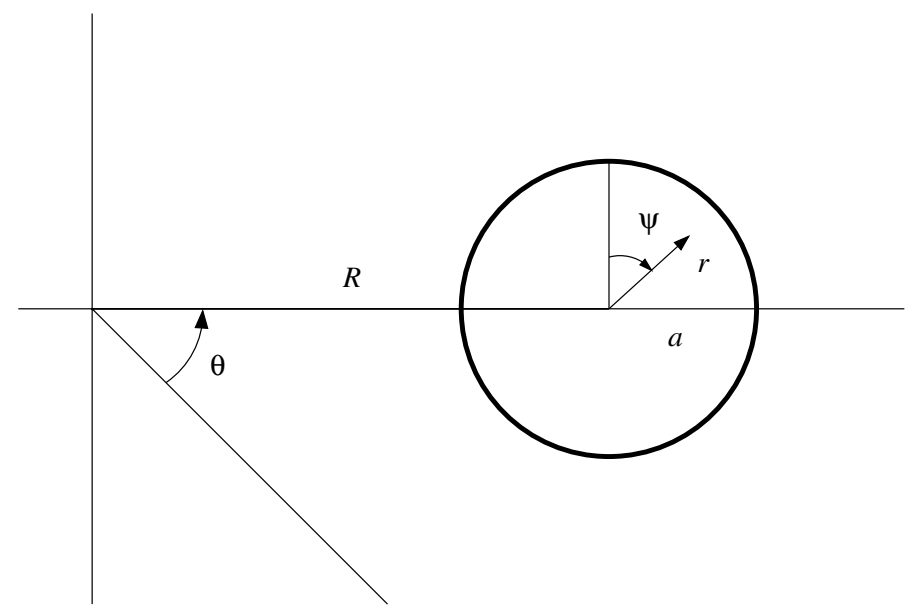

Figure 1: Cross section of the tube (inner wall at the left, outer wall at the right) and toroidal coordinates $(r, \psi, \theta)$.

\section{Unsteady solution}

By eqns. (4) and (5), the unsteady solution satisfies the following equation:

$$
\frac{\partial \hat{c}}{\partial t}+\overline{\boldsymbol{v}} \cdot \nabla \hat{c}+\hat{\boldsymbol{v}} \cdot \nabla \bar{c}-\mu \nabla^{2} \hat{c}=0
$$

with an homogeneous boundary condition at the wall:

$$
\mu \nabla \hat{c} \cdot \boldsymbol{n}+\sigma \hat{c}=0
$$

Because of eqns. (6)-(7), the homogeneous boundary value problem (8)-(9) depends only from the steady fluid velocity $\overline{\boldsymbol{v}}$ and is independent of the unsteady flow field $\hat{\boldsymbol{v}}$ and of the steady concentration part $\bar{c}$. A normalization criterion should be devised to select one solution of B.V.P. (8)-(9).

Let us now fix a precise shape to solve the problem (8)-(9). Let us consider a tube with the form of a long cylinder which may have a small degree of curvature, with the shape of a torus. This has a planar axis, a circular cross section of radius $a$ and constant radius of curvature $R$. The small deformation of the walls which is demonstrated of much importance in vascular dynamics [6], is irrelevant in the solute motion. For the following analysis, it is convenient to work out the equations in a toroidal coordinate system $(r, \theta, \psi)$ (fig. 1). The axial coordinate $z=R \theta$ is introduced to avoid degeneracy when $R \rightarrow \infty$ (straight tube).

The problem is now rewritten in nondimensional form by the following substitutions:

$$
\boldsymbol{x} \rightarrow \frac{\boldsymbol{x}}{a} \quad t \rightarrow \frac{V t}{a} \quad \boldsymbol{v} \rightarrow \frac{\boldsymbol{v}}{V}
$$

where $\boldsymbol{x}$ denotes the spatial coordinates and $V$ is a characteristic velocity. 
Without loss of generality, the concentration is considered dimensionless. By indicating by:

$$
P e=\frac{a V}{\mu} \quad \text { (Péclet number); } \quad S h=\frac{a \sigma}{\mu} \quad \text { (Sherwood number) }
$$

two characteristic numbers, the governing eqns. (8)-(9) become:

$$
\begin{aligned}
& \frac{\partial \hat{c}}{\partial t}+\overline{\boldsymbol{v}} \cdot \nabla \hat{c}-\frac{1}{P e} \nabla^{2} \hat{c}=0 \\
& \nabla \hat{c} \cdot \boldsymbol{n}+\operatorname{Sh} \hat{c}=0
\end{aligned}
$$

The physiological and metabolic functions of living beings are typically periodic and an intermittent release of substances (i.e. oxygen, hormones, nutrients, waste products) in the blood is secured by several organs and glands. Digestive and respiratory acts are also based over a periodical time scale and, according to the species, period can range from seconds to hours. It is realistic to assume that, for each substance, there exists a pulsatile source of solute concentration which, advected by the fluid, propagates downstream. Being the blood flow essentially unidirectional, the unsteady component $\hat{c}$ has the form of an harmonic longitudinal travelling wave:

$$
\hat{c}=\tilde{c}(r, \psi) e^{i(\omega t-k z)}
$$

with $\omega$ a nondimensional circular frequency $\left(\omega \rightarrow \frac{\omega a}{V}\right)$ and $k$ the nondimensional wave number $(k \rightarrow k a)$. In physiological cases, $\omega$ is generally very low $(\omega \ll 1)$.

\section{Perturbation solution}

All arteries are affected by a small or moderate degree of curvature. A perturbation method is used to study the influence of a small curvature with respect to the straight case. As the curvature parameter $\varepsilon=\frac{a}{R}$ is assumed to be small $(\ll 1)$, the amplitude in eqn. (12) is expanded as a power series of $\varepsilon$ over an axisymmetric solution $c_{0}(r)$. By omitting the $\sim$ sign at the right hand side, we have:

$$
\tilde{c}(r, \psi)=c_{0}(r)+\varepsilon c_{1}(r, \psi)+\varepsilon^{2} c_{2}(r, \psi)+\ldots
$$

The fluid steady velocity $\overline{\boldsymbol{v}}$ undergoes a similar expansion over $\overline{\boldsymbol{v}}_{\mathbf{0}}$ (the Poiseuille velocity) yielding:

$$
\overline{\boldsymbol{v}} \cdot \nabla \tilde{c}=\left(\overline{\boldsymbol{v}}_{0}+\varepsilon \overline{\boldsymbol{v}}_{1}\right) \cdot\left(\nabla c_{0}+\varepsilon \nabla c_{1}\right)=\overline{\boldsymbol{v}}_{0} \cdot \nabla c_{0}+\varepsilon\left(\overline{\boldsymbol{v}}_{1} \cdot \nabla c_{0}+\overline{\boldsymbol{v}}_{0} \cdot \nabla c_{1}\right)+\varepsilon^{2} \ldots
$$

Expression (12) and expansions (13)-(14) are substituted in eqn. (11), and terms of the same power of $\varepsilon$, up to the first order, are equated. 


\subsection{0-th order solution}

The amplitude of concentration in a straight tube is governed by the following linear equation:

$$
i \omega c_{0}+\overline{\boldsymbol{v}}_{\mathbf{0}} \cdot \nabla c_{0}-\frac{1}{P e} \nabla^{2} c_{0}=0
$$

Letting $\omega_{P}=\omega P e$ (scaled frequency) and $k_{P}=k P e$ (scaled wavenumber), eqn.(15) is rewritten in scalar notations as:

$$
\frac{d^{2} c_{0}}{d r^{2}}+\frac{1}{r} \frac{d c_{0}}{d r}+i\left(k_{P} \bar{w}_{0}-\omega_{P}\right) c_{0}=0
$$

where all terms containing $k^{2}$ have been neglected, since large wavelengths are considered in the present application, and

$$
\bar{w}_{0}(r)=1-r^{2}
$$

is the Poiseuille axial velocity profile, nondimensionalized by scaling with $V$.

The boundary conditions associated to the eqn. (16) are:

$$
\left.\frac{d c_{0}}{d r}\right|_{r=0}=0 \quad \frac{d c_{0}}{d r}+\left.S h c_{0}\right|_{r=1}=0
$$

For a given frequency $\omega_{P}$, the Sturm-Liouville eigenvalue problem (16)-(17) is solved to obtain the wave number $k_{P}$ which corresponds to an admissible $c$-wave solution in a straight tube. A normalization is assigned as an integral condition to specify a mass flux $Q$ :

$$
\int_{0}^{1} c_{0}(r) r d r=Q
$$

\subsection{1st order solution}

The correction due to a small curvature is described by the first order linear problem:

$$
i \omega c_{1}+\overline{\boldsymbol{v}}_{\mathbf{0}} \cdot \nabla c_{1}-\frac{1}{P e} \nabla^{2} c_{1}=-\overline{\boldsymbol{v}}_{\mathbf{1}} \cdot \nabla c_{0}
$$

By letting:

$$
c_{1}(r, \psi)=\check{c}_{1}(r) \sin \psi \quad \check{c}_{1} \rightarrow c_{1}
$$

we obtain the non-homogeneous problem:

$$
\frac{\partial^{2} c_{1}}{\partial r^{2}}+\frac{1}{r} \frac{\partial c_{1}}{\partial r}-\frac{c_{1}}{r^{2}}+i\left(k_{P} \bar{w}_{0}-\omega_{P}\right) c_{1}
$$




$$
=i k_{P}\left(r \bar{w}_{0}-w_{d}\right) c_{0}+\left(P e u_{d}-1\right) \frac{d c_{0}}{d r}
$$

with the boundary conditions:

$$
\left.c_{1}\right|_{r=0}=0 \quad \frac{d c_{1}}{d r}+\left.S h c_{1}\right|_{r=1}=0
$$

where $u_{d}$ and $w_{d}$ are respectively the nondimensional radial and the axial component of the steady flow in a curved tube [9]. Note that the Péclet number appears at the right hand side of eqn. (20) as coefficient of $u_{d}$. The solution turns out to be strongly dependent on it, because it magnifies the role of secondary flow. Such effect exists as long as a transverse flow, induced by the curvature, is present, and grows with $P e$.

Note that, due to the antisymmetry of the first order solution $c_{1}$ (see eqn. (19)), the overall mass flux conservation of $c_{0}+\varepsilon c_{1}$ in the half-section $(r, \psi) \in[0,1] \times$ $[-\pi / 2, \pi / 2]$ is guaranteed.

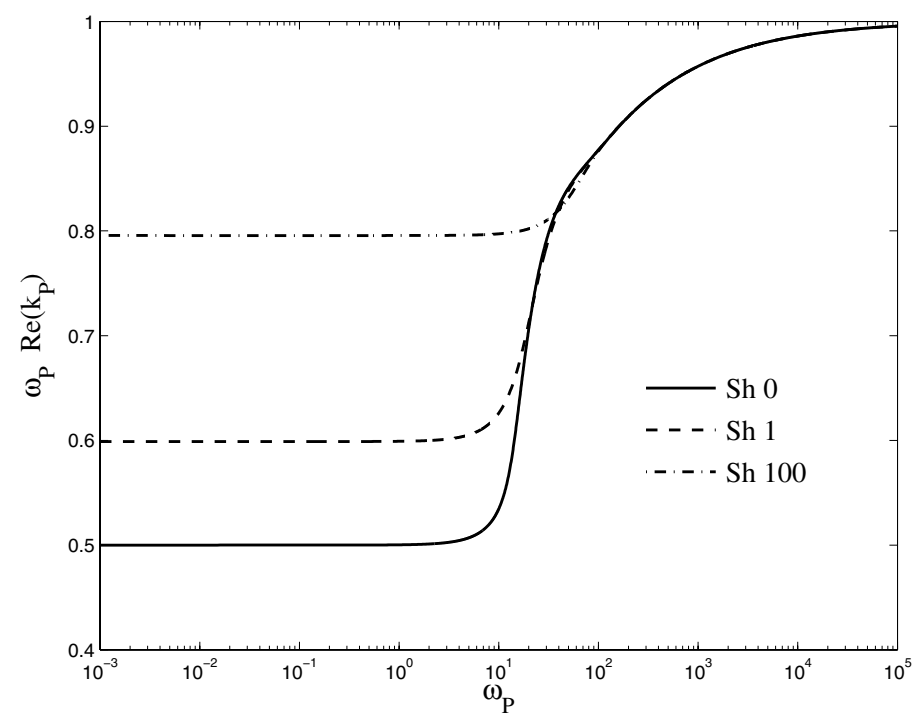

Figure 2: Dispersion curves for three Sherwood numbers. The wave speed tends to the same asymptotic value for relatively high frequencies and exhibits a variation with $S h$ only at low frequencies. In a range of typical frequency $\left(10 \leq \omega_{P} \leq 100\right)$, the speed undergoes a sudden raising. 


\section{Numerical method and parameters}

Firstly, we solved the Sturm-Liouville eigenvalue problem (16)-(17) in correspondence of a frequency $\omega_{P}$. This has been made numerically with a collocation method using a cubic spline approximating function [10]. The second-order differential problem is firstly reformulated as a system of two first-order ODE's. By means of an iterative procedure, the mesh is adapted in order to control the size of the residue and an initial guess is given by a quadratic polynomial satisfying the boundary conditions.

The method is fourth order accuracy and the algorithm is implemented through the routine bvp $4 \mathrm{C}$ of MATLAB. Since it uses a closed integration formula, a simple recipe to avoid the singularity at 0 has been devised. The solution has been normalized using eqn. (18).

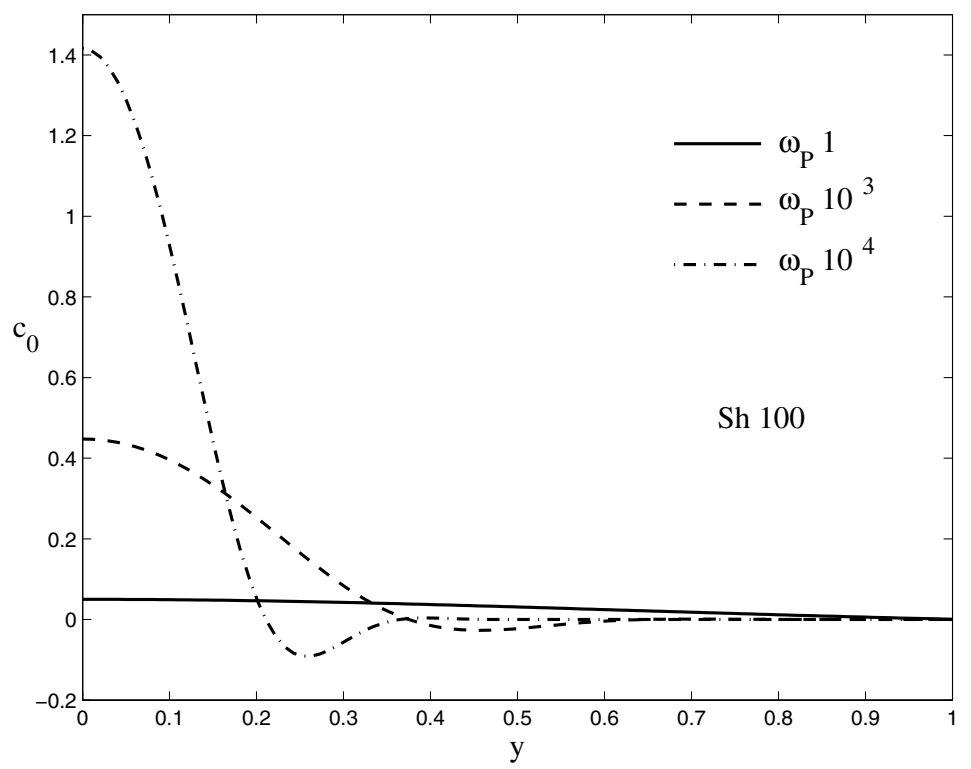

Figure 3: Concentration profiles $c_{0}$ along the horizontal half-diameter $(\psi=\pi / 2)$ of the cross section $z=0$ at $t=0$, for three values of $\omega_{P}$. A core flux is evident at higher frequencies.

Since we are interested to compute the solution for a set of frequencies, and being the problem sensitive to the initial guess, a chain of continuation steps along the path $\left(\omega_{P}, c_{0}\left(\cdot, \omega_{P}\right)\right)$ is initiated from $\left(0, c_{0}(\cdot, 0)\right)$. At each step, the solution just obtained for $\omega_{P}$ is used as initial guess for solving the same problem with a subsequent close value $\omega_{P}+\Delta \omega_{P}$ (continuation process). 
Thus, we construct a set of pairs $\left(\omega_{P}, \frac{\omega_{P}}{\operatorname{Re}\left(k_{P}\right)}\right)$ for $\omega_{P} \in\left[10^{-3}, 10^{5}\right]$, expressing the admissible solutions (dispersion curve, see fig. 2). It turns out that the wave speed tends to the asymptotic value 1 (independently of $S h$ ), for relatively large values of the scaled frequency. On the other hand, at very small frequencies the wave speed is rapidly decreasing tending to a finite limit as $\omega_{P} \rightarrow 0$. Such limit is $1 / 2$ for $S h=0$, and increase with $S h$ (fig. 2). A critical frequency separates two regimes for each value of $S h$ : a layer where the velocity undergoes a sudden raise from a larger range where the velocity stays almost constant.

The full wave solution is reassembled (see eqns. (3), (12) and (13)) as:

$$
c=\bar{c}+\tilde{c} e^{i(\omega t-k z)}=\bar{c}+\left(c_{0}+\varepsilon c_{1} \sin \psi\right) e^{i(\omega t-k z)}
$$
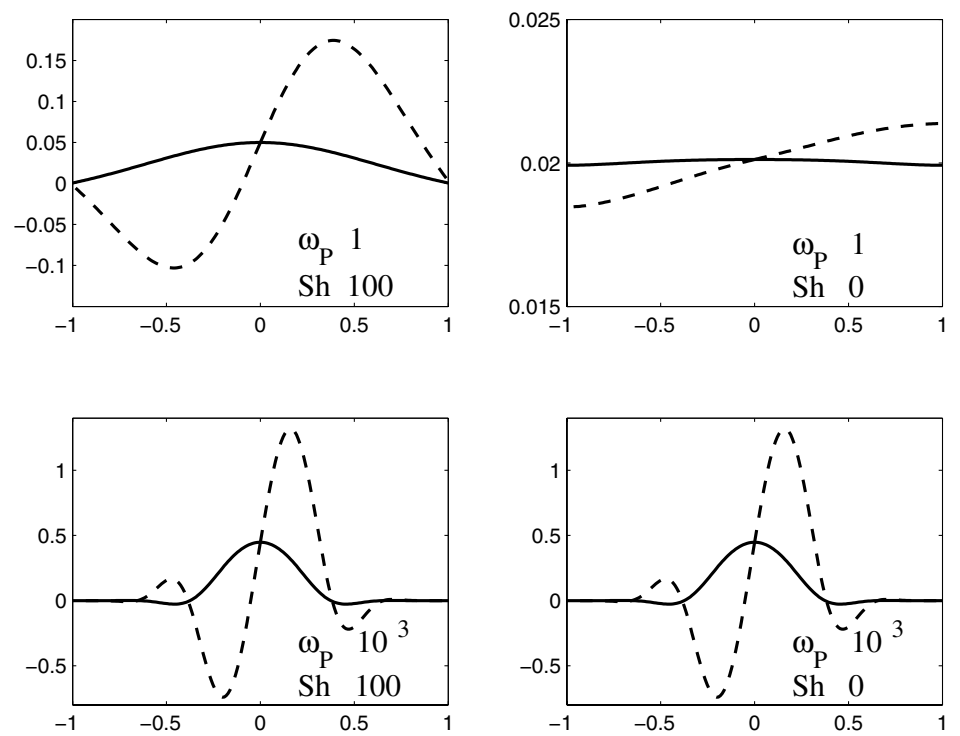

Figure 4: Concentration profiles along the horizontal diameter $(\psi= \pm \pi / 2)$ of the cross section $z=0$ at $t=0$ for $P e=10^{5}$. Plots highlight the combined effects of the wall permeability $S h$ (left-right) and of the wave frequency $\omega_{P}$ (top-bottom) in the case of a straight tube (continuous line) and of a slightly curved tube with $\varepsilon=10^{-4}$ (dashed line). For such value of $P e$, the solution is extremely sensitive to the curvature and, only at low frequency, even to $S h$.

An harmonic form of the previous expression is:

$$
c=\bar{c}+[\operatorname{Re}(\tilde{c}) \cos (\omega t-\operatorname{Re}(k) z)-\operatorname{Im}(\tilde{c}) \sin (\omega t-\operatorname{Re}(k) z)] e^{\operatorname{Im}(k) z}
$$


or equivalently:

$$
c=\bar{c}+|\tilde{c}| \cos (\omega t-\operatorname{Re}(k) z+\phi) e^{\operatorname{Im}(k) z} \quad \text { with } \phi=\arg (\tilde{c})
$$

The physical problem depends on a number of parameters, each of them may vary in a quite wide range, and there is a variety of different limiting cases. In the present work we will focus the attention on the influence of the solution $c$ on the diffusivity - parametrized by $P e$ - and on the wall permeability - parametrized by $S h$. These two parameters are varied in a convenient interval to describe a number of substances dissolved in blood and different medium properties.

The other parameters are fixed as:

$$
a=0.5 \mathrm{~cm} \quad V=24 \mathrm{cms}^{-1} \quad R e=300 \quad Q=0.01
$$

\section{Discussion and conclusions}

Concentration amplitudes $c_{0}$ for three typical values of $\omega_{P}$, with $S h=100$, are shown in fig. 3. Approximately flat concentration profiles at low $\omega_{P}$, are replaced by more oscillating fronts at higher $\omega_{P}$. At relatively higher $\omega_{P}$, the concentration flux occurs in the core of the vessel and is independent of Sh. A moderate dependence of $c$ on the wall permeability $S h$ exists at low frequencies.

The influence of curvature is small at low $\omega_{P}$, but becomes relevant at higher $\omega_{P}$, with a more pronounced oscillating profile. A noticeable difference with respect to a straight tube appears even for a curvature ratio small as $\varepsilon=10^{-4}$ (fig. 4). The first order solution $c_{1}$ is of few orders of magnitude higher than $c_{0}$, and their ratio grows with $\mathrm{Pe}$. It is extremely sensitive to the curvature and, at low frequency, even to $S h$.

A significant result is the skewness of the $c$ profiles: the maximum peak of concentration flux is shifted towards the outer bend and increases in magnitude. Such first order correction due to the curvature is expressed as:

$$
\Sigma(\varepsilon)=2 \varepsilon \int_{0}^{\frac{\pi}{2}} \sin \psi d \psi \int_{0}^{1} c_{1}(r) r d r=2 \varepsilon \int_{0}^{1} c_{1}(r) r d r
$$

The value of $\Sigma$ is extremely sensitive to the values of $R e$ and $P e$.

\section{References}

[1] Rappitsch G., Perktold K. \& Pernkopf E., Numerical modelling of sheardependent mass transfer in large arteries, Int. J. Num. Meth. Fluids, 25, pp. 847-857, 1997.

[2] Karner G., Perktold K., Zehentner H.P. \& Prosi M., Mass transport in large arteries and through the arterial wall, in Intra and Extracorporeal Cardiovascular Fluid Dynamics, Adv. Fluid Mech., P. Verdonck, K. Perktold eds. WIT press, pp. 209-247, 2000. 
[3] Prosi M., Perktold K., Ding Z. \& Friedman M.H., Influence of curvature dynamics on pulsatile coronary artery flow in a realistic bifurcation model, $J$. Biomech., 37, pp. 1767-1775, 2004.

[4] Quarteroni A., Veneziani A. \& Zunino P., Mathematical and numerical modeling of solute dynamics in blood flow and arterial walls, SIAM, J. Num. Anal., 39(5), pp.1488-1511, 2002.

[5] Moore J.A. \& Ethier C.R., Oxygen mass transfer calculations in large arteries, Jour. Biomech. Engin., 119, pp. 469-475, 1997.

[6] Pontrelli G. \& Tatone A., Wave propagation in a fluid flowing through a curved thin-walled elastic tube, Euro J. Mech., submitted, 2004.

[7] Bird R.B., Stewart W.E. \& Lightfoot E.N., Transport phenomena, John Wiley \& Sons, 1960.

[8] Caro C.G., Pedley T.J., Schroter R.C. \& Seed W.A., The mechanics of the circulation, Ch. 9, Oxford Univ. Press, 1978.

[9] Dean W.R., Note on the motion of fluid in a curved pipe, Phil. Mag., 4, pp. 208-223, 1927.

[10] Pryce J.D., Numerical solution of Sturm-Liouville problems, Monographs on Numerical Analysis, Oxford Univ. Press, 1993. 